\title{
Editorial: Electronics and Optoelectronics of Graphene and Related 2D Materials
}

\author{
Xinming $\mathrm{Li}^{1 *}$, Xu Zhang ${ }^{2 *}$, Hyesung Park ${ }^{3 *}$ and Antonio Di Bartolomeo ${ }^{4 *}$ \\ ${ }^{1}$ Guangdong Provincial Key Laboratory of Nanophotonic Functional Materials and Devices, School of Information and \\ Optoelectronic Science and Engineering, South China Normal University, Guangzhou, China, ${ }^{2}$ Department of Electrical and \\ Computer Engineering, Carnegie Mellon University, Pittsburgh, PA, United States, ${ }^{3}$ Department of Materials Science and \\ Engineering, Low Dimensional Carbon Materials Center, Perovtronics Research Center, Ulsan National Institute of Science \\ and Technology (UNIST), Ulsan, South Korea, ${ }^{4}$ Physics Department "E. R. Caianiello," University of Salerno, Fisciano, Italy
}

Keywords: graphene, 2D materials, optoelectronics, electronics, sensors

\section{Editorial on the Research Topic}

\section{Electronics and Optoelectronics of Graphene and Related 2D Materials}

The development of electronic and optoelectronic devices is often accompanied by the breakthroughs in semiconductor materials and device design. In recent years, layered atomic materials represented by graphene and other two-dimensional (2D) materials have received extensive attention due to their electronic and optical properties that are different from bulk materials. Based on graphene and 2D materials, the related physical mechanisms in electronics and optoelectronics can be explored. It is for this purpose that we have edited this Research Topic and look forward to summarizing the recent developments in this field. The four articles in this Research Topic involve the preparation of graphene nanosheets by liquid-phase exfoliation, the chemical vapor deposition (CVD) growth of graphene, the recent research progress of waveguide-integrated graphene photonic devices, and the mode-locked fiber laser modulated by $\mathrm{PtSe}_{2}$. In the next step, we highlight the important progress and related discussions involved in this Research Topic.

The preparation of $2 \mathrm{D}$ materials by liquid-phase exfoliation is a process of converting bulk materials into nanosheets by overcoming the van der Waals forces between the nanosheet layers (Ciesielski and Samorì, 2014). Owing to the relatively easy process, low cost, short preparation time, and large-scale manufacturing, liquid-phase exfoliation is currently considered one of the most promising production techniques that can promote the industrial applications of graphene and 2D materials, such as printed electronics, composite fillers and conductive coatings (Phiri et al., 2017; Pang et al., 2019; Di Bartolomeo et al., 2020). In this Research Topic, (Li et al.) summarize the mechanisms and methods for graphene liquid-phase exfoliation and stable dispersion. The liquid exfoliation mechanism, in which a dispersant-solvent system interacts with the graphene surface suppressing the van der Waals forces between graphene layers, is discussed using the Hamaker constant theory (Coleman, 2013). The main liquid-phase exfoliation methods, such as ultrasonic degradation, mechanical exfoliation, and electrochemical exfoliation are analyzed in detail. On the other hand, the ultimate aim of liquid phase exfoliation is a stable dispersion in various liquid media, suitable for practical applications. As graphene sheets tend to stick to each other, a stable dispersion requires an appropriate dispersant-solvent system that interacts with the graphene surface preventing graphene agglomeration. It has been experimentally shown that the best liquid-phase dispersion is accomplished when the surface tension of the dispersant-solvent system is equal to the surface tension of graphene (Vallés et al., 2008). Hence, the potential energy of different dispersant-solvent systems and their relationship with the energy of the graphene surface have been studied and several experimental 
approaches are discussed to show how the Hansen solubility coefficient theory ( $\mathrm{Lim}$ et al., 2014) has been the basis for selecting the dispersant-solvent system best suited to interact with graphene. Meanwhile, it has been shown that when the $\pi-\pi$ interaction of graphene with aromatic molecules becomes stronger, the liquid-phase dispersion properties of graphene improve (Georgakilas et al., 2016). Pyrene derivatives, biomolecules, carbon nanotubes, graphene oxide, and polymers have been introduced in liquid phase as the dispersant to enable $\pi-\pi$ interaction and attain graphene dispersion stability of several months. The formation of intermolecular hydrogen bonds is also profitable to the dispersion and dissolution of graphene. Oxygen-containing groups can remain on the surface of graphene prepared by oxidation-reduction methods and can be exploited to produce strong hydrogen bond with the dispersant-solvent system to achieve a stable dispersion of graphene. Hydrogen bond interaction has also been widely used as an alternative dispersion approach.

In addition to the liquid-phase exfoliation method, chemical vapor deposition (CVD) technology is another common method for achieving large-area graphene films. To fully unleash the potential of graphene, it is critical to advance the understanding of defect formation during CVD synthesis and find a way to effectively minimize the nucleation density. Introducing oxygen is able to increase the growth rate of graphene domains as an effective copper surface passivator, and transform the synthesis kinetics from edge-attachment-limited to diffusionlimited. Various techniques have been explored to introduce oxygen during graphene CVD growth, including injecting pure $\mathrm{O}_{2}$ gas into the reaction chamber (Hao et al., 2013), injecting a trace amount of $\mathrm{H}_{2} \mathrm{O}$ gas (Guo et al., 2018), annealing copper in an Ar environment for minimum oxidization (Li et al., 2015) and baking copper on a hot plate in the air (Ding et al., 2017). In this research article, Zhang et al. investigated the formation mechanism of graphene domain growth on pre-oxidized copper substrates via an atmospheric pressure chemical vapor deposition (APCVD) method. The authors did a systematic study on the evolution of the substrate surface during the annealing process in an $\mathrm{Ar} / \mathrm{H}_{2}$ reducing atmosphere. A lower nucleation density is desirable as it allows more space for the extended growth of a single domain. This paper shows that the domain size and nucleation density can be controlled by varying the annealing time. Transmission electron microscopy (TEM) was also used to investigate the crystalline nature of graphene domains. Besides, the gas ratio and temperature were also found to play an important role in the graphene domain growth. By optimizing reaction temperature and extending the growth time to 60 minutes can lead to a domain size with a lateral dimension of $\sim 720 \mu \mathrm{m}$ (Zhang et al.). This work advances the understanding of CVD growth kinetics of the graphene domain on the copper substrate, which is important for further optimization of highquality and large-area graphene films synthesis.

On the other hand, graphene and 2D materials based optoelectronic and photonic devices have attracted great interest in imaging, sensing and communication applications (Phare et al., 2015; Cheng and Goda, 2016; Chen et al., 2017; Tong et al., 2020). Due to its linear band structure and vanishing density of states at the Dirac point, graphene has a broad spectral band and tunable light absorption from visible to mid-infrared wavelengths. Meanwhile, the local carrier density of graphene can be easily changed to adjust its electrical and optical properties (Bonaccorso et al., 2010). However, due to the weak light absorption of single-layer graphene, the light-matter interaction is insufficient. In recent studies, the integration technology of graphene and photonic structures has been widely reported to enhance the light-matter interaction in graphene-based photonic devices (Fang et al., 2012; Li et al., 2012). In this Research Topic, Wang et al. summarized the principle and technical development of a graphene photonic device based on optical waveguide technology. In this device, the light-matter interaction in waveguide-integrated graphene photonic devices can be enhanced via the evanescent field coupling. Due to the adjustable conductivity of graphene, this waveguide-integrated graphene photonic devices can be used in the field of molecular detection sensors. Through the doping effect of adsorbed molecules on graphene, the functionality of waveguide-integrated graphene photonic devices will change, which can be used for the molecular sensors (Cheng and Goda, 2016). Besides, optically tunable graphene can be used for light modulation and detection. The waveguide-integrated graphene modulators can be divided into electro-absorption modulators and electro-refractive modulators (Phare et al., 2015; Sorianello et al., 2018). And waveguideintegrated graphene photodetectors can be achieved, in which the light absorption of graphene can be enhanced by the evanescent field interaction (Gan et al., 2013; Pospischil et al., 2013; Wang et al., 2013). Based on this mechanism, important device designs and methods are introduced in-depth in this Review article. In addition to the progress in the field of graphene-based optical waveguides, another article focuses on the progress of $\mathrm{PtSe}_{2}$ as saturable absorbers in mode-locked fiber lasers. Wu and Jiang studied the soliton formation, and observed the different soliton patterns by increasing the pump power ( $\mathrm{Wu}$ and Jiang). This study determines the nonlinear optical response of $\mathrm{PtSe}_{2}$ and provides a reference for the study of soliton dynamics in fiber laser systems.

The Research Topic on Electronics and Optoelectronics of Graphene and Related 2D Materials contains two Reviews and two original papers. Of course, this is far from covering the scope of this topic, but these developments can help readers understand and think about the development in this field. Finally, as the guest editors, we would like to thank the authors for their contributions and the reviewers for their efforts, as well as the editors and publishers for their support.

\section{AUTHOR CONTRIBUTIONS}

All authors listed have made a substantial, direct and intellectual contribution to the work, and approved it for publication.

\section{FUNDING}

XL acknowledges the financial support from South China Normal University start-up fund and the Guangdong Provincial Key Laboratory of Nanophotonic Functional Materials and Devices. XZ acknowledges the support from Carnegie Mellon University. 


\section{REFERENCES}

Bonaccorso, F., Sun, Z., Hasan, T., and Ferrai, A. C. (2010). Graphene photonics and optoelectronics. Nat. Photon. 4, 611-622. doi: 10.1038/nphoton.2010.186

Chen, Z., Li, X., Wang, J., Tao,. L., Long, M., Liang, S., et al. (2017). Synergistic effects of plasmonics and electron trapping in graphene short-wave infrared photodetectors with ultrahigh responsivity. ACS Nano. 11: 430-437. doi: 10.1021/acsnano.6b06172

Cheng, Z., and Goda, K. (2016). Design of waveguide-integrated graphene devices for photonic gas sensing. Nanotechnology 27:505206. doi: 10.1088/0957-4484/27/50/505206

Ciesielski, A., and Samorì, P. (2014). Graphene via sonication assisted liquid-phase exfoliation. Chem. Soc. Rev. 43, 381-398. doi: 10.1039/C3CS60217F

Coleman, J. N. (2013). Liquid exfoliation of defect-free graphene. Acc. Chem. Res. 46, 14-22. doi: 10.1021/ar300009f

Di Bartolomeo, A., Iemmo, L., Urban, F., Palomba, M., Carotenuto, G., Longo, A., et al. (2020). Graphite platelet films deposited by spray technique on low density polyethylene substrates. Mater. Today Proc. 20, 87-90. doi: 10.1016/j.matpr.2019.09.086

Ding, D., Solís-Fernández, P., Yunus, R. M., Hibino, H., and Ago, H. (2017). Behavior and role of superficial oxygen in $\mathrm{Cu}$ for the growth of large single-crystalline graphene. Appl. Surface Sci. 408, 142-149. doi: 10.1016/j.apsusc.2017.02.250

Fang, Z., Wang, Y., Liu, Z., Schlather, A., Ajayan, P. M., Koppens, F. H. L., et al. (2012). Plasmon-induced doping of graphene. ACS Nano. 6, 10222-10228. doi: $10.1021 / \mathrm{nn} 304028 \mathrm{~b}$

Gan, X., Shiue, R.-J., Gao, Y., Meric, I., Heinz, T. F., Shepard, K., et al. (2013). Chip-integrated ultrafast graphene photodetector with high responsivity. Nat. Photon. 7, 883-887. doi: 10.1038/nphoton.2013.253

Georgakilas, V., Tiwari, J. N., Kemp, K. C., Perman, J. A., Bourlinos, A. B., Kim, K. S., et al. (2016). Noncovalent functionalization of graphene and graphene oxide for energy materials, biosensing, catalytic, and biomedical applications. Chem. Rev. 116, 5464-5519. doi: 10.1021/acs.chemrev.5b00620

Guo, W., Wu, B., Wang, S., and Liu, Y. (2018). Controlling fundamental fluctuations for reproducible growth of large single-crystal graphene. ACS Nano 12, 1778-1784. doi: 10.1021/acsnano.7b08548

Hao, Y., Bharathi, M. S., Wang, L., Liu, Y., Chen, H., Nie, S., et al. (2013). The role of surface oxygen in the growth of large single-crystal graphene on copper. Science 342, 720-723. doi: 10.1126/science.1243879

Li, H., Anugrah, Y., Koester, S. J., and Li, M. (2012). Optical absorption in graphene integrated on silicon waveguides. Appl. Phys. Lett. 101:111110. doi: 10.1063/1.4752435

Li, J., Wang, X.-Y., Liu, X.-R., Jin, Z., Wang, D., and Wan, L.-J. (2015). Facile growth of centimeter-sized single-crystal graphene on copper foil at atmospheric pressure. J. Mater. Chem. C 3, 3530-3535. doi: $10.1039 / \mathrm{C} 5 \mathrm{TC} 00235 \mathrm{D}$
Lim, H. J., Lee, K., Cho, Y. S., Kim, Y. S., Kim, T., and Park, C. R. (2014). Experimental consideration of the Hansen solubility parameters of as-produced multi-walled carbon nanotubes by inverse gas chromatography. Phys. Chem. Chem. Phys. 16:17466. doi: 10.1039/C4CP0 2319F

Pang, Y., Yang, J., Curtis, T. E., Luo, S., Huang, D., Feng, Z., et al. (2019). Exfoliated graphene leads to exceptional mechanical properties of polymer composite films. ACS Nano. 13, 1097-1106. doi: 10.1021/acsnano.8b0 4734

Phare, C. T., Daniel Lee, Y.-H., Cardenas, J., and Lipson, M. (2015). Graphene electro-optic modulator with $30 \mathrm{GHz}$ bandwidth. Nat. Photon. 9, 511-514. doi: $10.1038 /$ nphoton.2015.122

Phiri, J., Gane, P., and Maloney, T. C. (2017). General overview of graphene: production, properties and application in polymer composites. Mater. Sci. Eng. B 215, 9-28. doi: 10.1016/j.mseb.2016.10.004

Pospischil, A., Humer, M., Furchi, M. M., Bachmann, D., Guider, R., Fromherz, T., et al. (2013). CMOS-compatible graphene photodetector covering all optical communication bands. Nat. Photon. 7, 892-896. doi: 10.1038/nphoton.2013.240

Sorianello, V., Midrio, M., Contestabile, G., Asselberghs, I., Van Campenhout, J., Huyghebaert, C., et al. (2018). Graphene-silicon phase modulators with gigahertz bandwidth. Nat. Photon. 12, 40-44. doi: 10.1038/s41566-017-0 071-6

Tong, L., Huang, X., Wang, P., Ye, L., Peng, M., An, L., et al. (2020). Stable mid-infrared polarization imaging based on quasi-2D tellurium at room temperature. Nat. Commun. 11:2308. doi: 10.1038/s41467-020-1 6125-8

Vallés, C., Drummond, C., Saadaoui, H., Furtado, C. A., He, M., Roubeau, O., et al. (2008). Solutions of negatively charged graphene sheets and ribbons. J. Am. Chem. Soc. 130, 15802-15804. doi: 10.1021/ja80 $8001 \mathrm{a}$

Wang, X., Cheng, Z., Xu, K., Tsang, H. K., and Xu, J.-B. (2013). High-responsivity graphene/silicon-heterostructure waveguide photodetectors. Nat. Photon. 7, 888-891. doi: 10.1038/nphoton.2013.241

Conflict of Interest: The authors declare that the research was conducted in the absence of any commercial or financial relationships that could be construed as a potential conflict of interest.

Copyright (C) 2020 Li, Zhang, Park and Di Bartolomeo. This is an open-access article distributed under the terms of the Creative Commons Attribution License (CC BY). The use, distribution or reproduction in other forums is permitted, provided the original author(s) and the copyright owner(s) are credited and that the original publication in this journal is cited, in accordance with accepted academic practice. No use, distribution or reproduction is permitted which does not comply with these terms. 